\title{
Developmental changes of the central sulcus morphology in young children
}

Niharika Gajawelli ${ }^{1}$, Sean Deoni ${ }^{3,4,5}$, Natalie Ramsy ${ }^{2}$, Douglas C Dean III ${ }^{6,7,8}$, Jonathan O’Muircheartaigh ${ }^{9}$, Marvin D. Nelson ${ }^{1,10}$, Natasha Lepore ${ }^{1,10 *}$ and Olivier Coulon ${ }^{11} *$ [* equal senior author contribution]

Corresponding author: Natasha Lepore nlepore@chla.usc.edu

1. CIBORG Laboratory, Department of Radiology, Children's Hospital of Los Angeles Address: 4650 Sunset Blvd, Los Angeles, CA, 90027 ,

Phone: +1 323-361-2411

2. Carle Illinois College of Medicine

Address: 807 S Wright St, Champaign, IL 61820

Phone: +1 213 740-7237

3. Advanced Baby Imaging Lab, Hasbro Childrens Hospital, Providence Address: 593 Eddy Street Ground Level, Providence, RI 02903

Phone: +1 (401) 444-7770

4. Pediatrics and Radiology, Warren Alpert medical school, Brown University Address: 222 Richmond St, Providence, RI 02903

Phone: 401-863-1000

5. Maternal, Newborn \& Child Health Discovery \& Tools at the Bill and Melinda Gates Foundation Address: 500 5th Ave N, Seattle, WA 98109

Phone: $+1 \underline{(206) 709-3100}$

6. Waisman Laboratory for Brain Imaging and Behavior, Waisman Center, University of Wisconsin-Madison Address: 1500 Highland Ave, Madison WI 53705

Phone: +1 608-263-1656

7. Department of Pediatrics, University of Wisconsin School of Medicine and Public Health 600 Highland Ave, Madison, WI 53792

Phone: +1 (608) 262-8785

8. Department of Medical Physics, University of Wisconsin School of Medicine and Public Health Address: 750 Highland Ave. Madison, WI 53705

Phone: +1 608-263-4900

9. Department of Neuroimaging, King's College London Address: 2nd Floor, Centre for Neuroimaging Science, Denmark Hill, London, United Kingdom Phone: +44 02078385162

10. Department of Radiology, Keck School of Medicine, University of Southern California Address:1500 San Pablo Street, Los Angeles, CA 90033

Phone: +1 800-872-2273

11. Institut de neurosciences de la Timone, Aix-Marseille Univ, CNRS UMR7289, France Address: Faculty of Medicine, 27, boulevard Jean Moulin, 13005 Marseille - France Phone: +33 (0) 491324059 


\section{Developmental changes of the central sulcus morphology in young children}

Niharika Gajawelli, Sean Deoni, Natalie Ramsy, Douglas Dean, Jonathan O'Muircheartaigh, Marvin D. Nelson, Natasha Lepore* and Olivier Coulon*

[* equal senior author contribution]

Abstract — "The human brain grows rapidly in early childhood, reaching 95\% of its final volume by age

6. Understanding brain growth in childhood is important both to answer neuroscience questions about anatomical changes in development, and as a comparison metric for neurological disorders. Metrics for neuroanatomical development including cortical measures pertaining to the sulci can be instrumental in early diagnosis, monitoring and intervention for neurological diseases. In this paper, we examine the development of the central sulcus in children aged 12 to 60 months from structural magnetic resonance images. The central sulcus is one of the earliest sulci to develop at the fetal stage and is implicated in diseases such as Attention Deficit Hyperactive Disorder and Williams syndrome. We investigate the relationship between the changes in the depth of the central sulcus with respect to age. In our results, we observed a pattern of depth present early on, that had been previously observed in adults. Results also reveal the presence of a rightward depth asymmetry at age 12 month at a location related to orofacial movements. That asymmetry disappears gradually, mostly between 12 and 24 months, and we suggest that it is related to the development of language skills."

\section{INTRODUCTION}

Normal development of the brain is of key interest in both neuroscience and medicine. In the human brain, different tissue types, brain structures and neural circuits have distinctive developmental patterns throughout the lifespan. Various features of cortical development in childhood have been explored as part of lifespan studies in previous publications (Sowell et al., n.d.; 2003; Toga, Thompson, and Sowell 2011; S. Li et al. 2011; Courchesne et al. 2000; Nie, Li, and Shen 2013; Giedd and Rapoport 2010; Sowell et al. 2007; Brain Development Cooperative Group 2012; Lenroot and Giedd 2006; Raznahan et al. 2011; 
Keshavan et al. 2002; Evans 2006). Longitudinal MR studies investigating brain development have shown inverted $U$ shaped trajectories of gray matter (GM) volumes and that these trajectories vary widely over different regions of the cortex (Giedd and Rapoport 2010; Lenroot and Giedd 2006; Sowell et al. 2003; Raznahan et al. 2011; Knickmeyer et al. 2008). Age-related decreases in lobar gray matter were reported most prominent in the parietal and occipital cortex in children between the ages of 4.5-18 years of age (Brain Development Cooperative Group 2012). In early childhood, a longitudinal study of regional cortical thickness and surface area in healthy infants from birth to 2 years showed that cortical thickness, by age 2 , is on average $97 \%$ of adult values, while surface area is $69 \%$, suggesting that surface area expansion is a driving factor in cortical volume changes after age 2 (Lyall et al. 2015).

Cortical folding, a lesser explored feature of brain development, begins at about 20 weeks gestational age (Gilles and Nelson 2012), and keeps evolving through childhood (Andersen et al., n.d.). Gyri and sulci parameters can be affected by genetics and environmental factors, however, consistent similarity exists in the fold patterns and sulci of healthy individuals (Coulon et al. 2015)'(Li et al. 2015). Hence, understanding sulcal and gyral growth can provide valuable insight into brain development (Li et al. 2014a). Studies on cortical folding development done so far have typically had a small set of subjects (Li et al. 2014b) and a limited age range. The smaller subsets of data make it challenging to account for variability in brain development between subjects. While such studies have investigated cortical folding or gyrification patterns over the entire brain in infancy (Li et al. 2014a; Hill et al. 2010), there still remain many parameters of the brain to be investigated, such as sulcal depth, width, curvature as well as developmental trajectories of different brain regions over a longer duration.

Due to the rapid cerebral changes and environmental influences that contribute to the learning of new skills in young children, the central sulcus (CS), a prominent sulcus that begins its development in the fetal stage, is a promising biological trait to investigate in development. The CS lies within the paracentral gyrus and in between the pre and postcentral gyri, corresponding to the motor and somatosensory cortices respectively and is highly involved in somatosensory and motor function The CS depth is of particular interest due to its variation through its length and the association with handedness 
(Amunts et al. 2000; 1996; Cykowski et al. 2008). The CS contains a distinct fold that connects the pre and postcentral gyri, termed by Broca as the "pli de passage fronto-parietal moyen" or PPFM (W. W. Boling and Olivier 2004; W. Boling et al. 1999), where hand motor function has shown to be localized (W. W. Boling and Olivier 2004), and also where the hand knob (Yousry et al. 1997) is located. While the area on both sides of the PPFM has been shown to be highly heritable, the PPFM itself did not show genetic correlations (McKay et al. 2013), postulating the association with plasticity, driven by environmental factors. CS depth and subtle abnormalities associated with it can also be a marker of functional specificities or developmental pathologies (G. Li, Nie, et al. 2014b; Cykowski et al. 2008; Jackowski and Schultz 2005; Galaburda et al. 2001), such as in Williams syndrome and ADHD (S. Li et al. 2015). Therefore, in order to gain insight into why and how the CS morphometry differs between populations, it is important to establish a baseline of normal cortical folds maturation.

In this paper, we focus specifically on the changes that occur in the CS in early childhood in normal development between 12 to 60 months of age. Through analysis of CS depth curves, we study typical developmental changes of CS morphology in a cross-sectional dataset and investigate regions of maximum change. To our knowledge, no previous studies have investigated specifically individual sulcal measures in healthy childhood development. Hence, results may clarify the specificity of changes that occur during early childhood. Also, while out of the scope of this paper, similar investigations for each sulcus may lead to potential applications as anatomical biomarkers for disease (Galaburda et al. 2001; Jackowski and Schultz 2005)

\section{MATERIAL AND METHODS}

\section{A. Data}

We selected brain MR volumes from 111 subjects ranging in age from 270 to 1900 days (roughly corresponding to 12 months to 60 months) from the Advanced Baby Imaging Lab database (www.babyimaginglab.com). The data was largely cross-sectional and was processed as such. Only 17 of 
the 111 subjects had several time points. 10 male and 5 female subjects had scans at 2 time points, and 2 female subjects had scans at 3 time points. This resulted in a set of 130 MR images.

The data used consisted of T1 MP-RAGE MRI scans $\left(1.4-1.8 \mathrm{~mm}^{3}\right)$ of healthy normal children with the following inclusion criteria: singleton birth between 37 and 42 weeks gestation with no abnormalities on fetal ultrasound and no reported history of neurological events or disorders in the infant. Data acquisition details can be found in (Deoni, Rutt, and Peters 2003; 2006). Each subject or their guardian was informed of the goals of the study and signed a formal consent. The study was approved by the Institutional Review Board of Brown University and informed consent was obtained from the guardians of all participants. All data was de-identified before pre-processing. Note that handedness of the subjects is not assessed in this study due to lack of handedness information at early ages.

\section{B. Processing}

Brain volumes were first skull-stripped using the BrainSuite (Shattuck and Leahy 2002) and linearly registered with 6 degrees of freedom to an age matched template using FSL FLIRT (M Jenkinson and Smith 2001; Mark Jenkinson et al. 2002). A geodesic intensity correction algorithm (Gaonkar et al. 2015) was then applied for bias field correction, and the volume was resampled to a $1 \times 1 \times 1 \mathrm{~mm}^{3}$ space. The geodesic bias field correction was used to improve the lower contrast between gray and white matter seen in children compared to adults, leading to a more accurate automatic gray/white tissue segmentation using BrainVisa morphologist pipeline (Fischer et al. 2012). The histogram of the gray/white matter was manually tuned to achieve an accurate brain mask and hemispheric split. Using the graphs containing cortical sulci meshes produced by BrainVisa (Perrot, Rivière, and Mangin 2011), we manually identified the CS and applied the sulcal parameterization pipeline to the left and right CS of each subject (Olivier Coulon et al. 2015), in a depth measure at each position along the sulcus. The sulcal parameterization process detects the dorsal and ventral extremities of the CS and computes a smooth isometric parameterization for the CS (see Fig. 1). This gives us a relative position between the two extremities for 
each point along the CS. The depth at each position between $[0,100]$ was computed by measuring the length of the corresponding isoparametric lines as shown in Figure 1.

In previous morphological statistical studies, the resulting sulcal depth profiles have shown good inter-subject reproducibility and have been used for group studies ${ }^{18,28,32,44,46 .}$ For calculation of the mean sulcal depth and group comparisons, the data was grouped into 6-month intervals for age groups from 12 to 24 months and 12-month intervals thereafter. This interval was chosen to retain statistical power as well as capture the changes in the younger age groups. Additionally, due to fewer subjects at 48 and 60 months (11 and 5 subjects respectively), and since growth was expected to be less rapid compared to the younger ages (Remer et al. 2017), these two cohorts were grouped together. The distribution of ages and number of subjects are shown in Table 1.

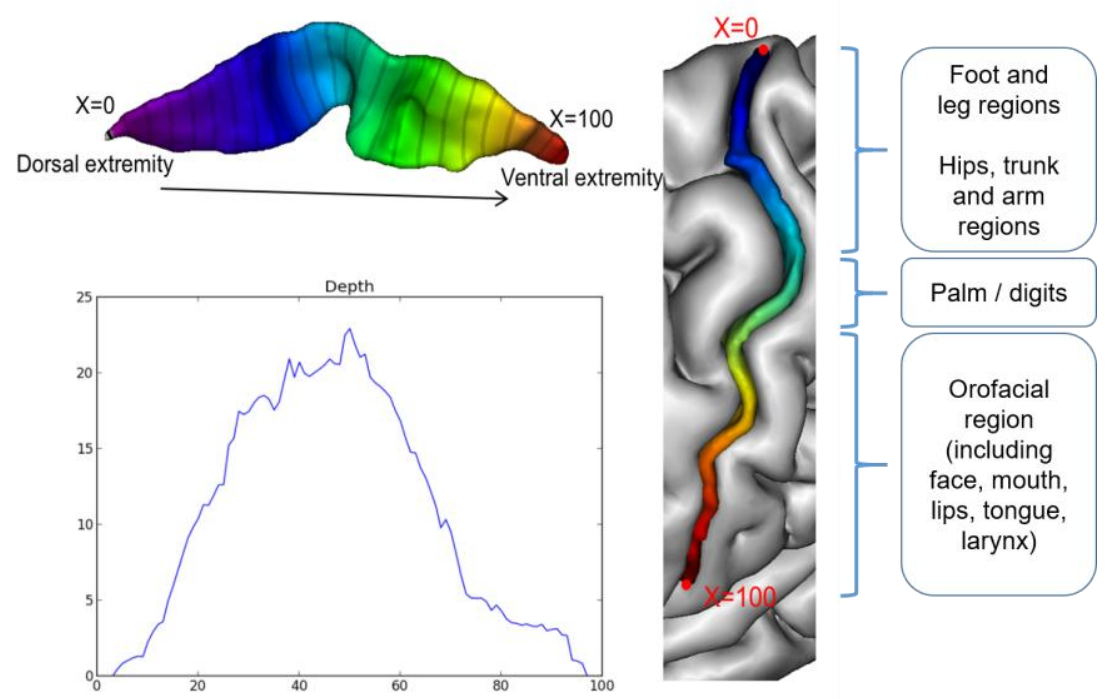

Figure 1. Central sulcus of an adult subject, with its parameterization and the corresponding depth profile. Approximate anatomical regions from literature (Catani 2017; Roux, Djidjeli, and Durand 2018) are listed for reference.

\begin{tabular}{|l|l|l|l|}
\hline Age & Male & Female & Total \\
\hline 12 months & 18 & 18 & 36 \\
\hline 18 months & 16 & 9 & 25 \\
\hline 24 months & 13 & 13 & 26 \\
\hline
\end{tabular}




\begin{tabular}{|l|l|l|l|}
\hline 36 months & 17 & 10 & 27 \\
\hline 48 and 60 months & 11 & 5 & 16 \\
\hline
\end{tabular}

Table 1. Table showing the number of scans in the different age groups

\section{Mean CS depth curves and regression}

First, the data was binned into groups as in Table 1 and the mean and standard deviation in sulcal depth for each group $(12,18,24,36$ and $48+60$ month groups) was computed. The mean depth curves were smoothed with a moving average span of 3. Since the CS depth is our feature of interest and has been related to functional localization (W. W. Boling and Olivier 2004; O. Coulon et al. 2011) and functional specificities such as handedness (Cykowski et al. 2008; Hopkins, Coulon, and Mangin 2010), regression was used to characterize the sulcal depth developmental trajectories at each of the 100 positions of the CS for all subjects. The following equations showing the linear, logarithmic and quadratic models that were tested:

$$
\begin{gathered}
\text { Linear: sulcal depth } \sim B 1 * \text { age }+B 2 \\
\text { Quaratic: sulcal depth } \sim B 1 * \text { age } e^{2}+B 2 * \text { age }+B 3 \\
\text { Logarithmic: sulcal depth } \sim B 1 * \ln (\text { age })+B 2
\end{gathered}
$$

The Bayesian Information Criterion (BIC) was computed for each model. The BIC values indicated the best fit as the logarithmic model for most of the 100 positions (87 points in the left hemisphere and 64 points in the right hemisphere), and therefore was used in for regression analysis in this paper. Significance was defined as $\alpha<0.0005$ ( $\mathrm{p}<0.05$ Bonferroni corrected for 100 positions along the CS).

\section{Group differences}


To compare the differences in CS depth between the 12 vs $18 \mathrm{~m}, 18$ vs 24 month, 24 vs 36 month, 36 vs 48+ month groups Mann Whitney tests were conducted at each of the 100 positions along the CS and then corrected for multiple comparisons via permutation testing. The Mann Whitney test was used to avoid assumption of a Gaussian distribution of the sulcal depth. Those group comparisons were done to identify positions of the CS of significant depth change between groups.

\section{Asymmetry calculation}

To characterize asymmetries in CS depth in both hemispheres, the asymmetry coefficients $[\mathrm{CA}=($ right CS depth - left CS depth)/0.5*(right CS depth + left CS depth)] were calculated for each point on the CS depth curve. Therefore, a negative value of the CA indicates leftward asymmetry. The CA at each position was compared to zero for each age group defined above using Mann-Whitney rank tests and then Bonferroni corrected. Logarithmic regression was used to identify where asymmetry change was significantly associated with age. Additionally, a sliding window approach with window size 50 was used to identify the ages where the most change was seen. Ordering the data by age from the youngest to oldest, the mean CA was averaged for the first 50 subjects. Subsequently, the mean CA was then averaged for the next 50 subjects from $2^{\text {nd }}$ youngest subject onwards and so on. Finally, for each of the 100 positions along the CS, a single sample t-test provided identification of significant windows.

\section{RESULTS}

The mean CS depth curves indicate similarity to the pattern seen in adults (Cykowski et al. 2008) identifiable as early as 12 months of age. A strong relationship was observed between sulcal depth and age in the left central sulcus in regions that correspond to the jaw, mouth and tongue regions, with the largest change seen between 18 and 24 months of age.

The mean sulcal depth curves of the left and right CS in age groups 12, 24. 36, and 48+ months are shown in Fig. 2. In all groups and in both hemispheres, there is a steep increase in the depth profiles 
from the superior part of the CS (position 1) to around position 32, followed by a small dip around position 50 with a gradual increase in the depth profile until the next peak at around position 65 , after which the curves decline steeply. These three features have been previously identified respectively as the superior peak, pli de passage fronto-parietal moyen (PPFM), and inferior peak (Cykowski et al. 2008). The dip corresponding to the PPFM is most prominent in the older children.

The CS depth followed predominantly logarithmic changes in most positions. Correlation coefficients found from the regression plotted against the sulcal positions in Fig. 3 revealed the significance in CS positions between 60-85 in the left hemisphere, corresponding to the mouth region as depicted on the motor and somatosensory homunculus and in positions between $30-45$ in the right hemisphere, corresponding to the upper torso, arms, and hands. The mean depth curve of all ages is also plotted as reference and the dots indicate significant points after Bonferroni correction. To further understand changes in these regions of significance, a post hoc correlation of age and CS depth was performed for the two most significant points and fitted with a logarithmic curve (Fig. 4). 

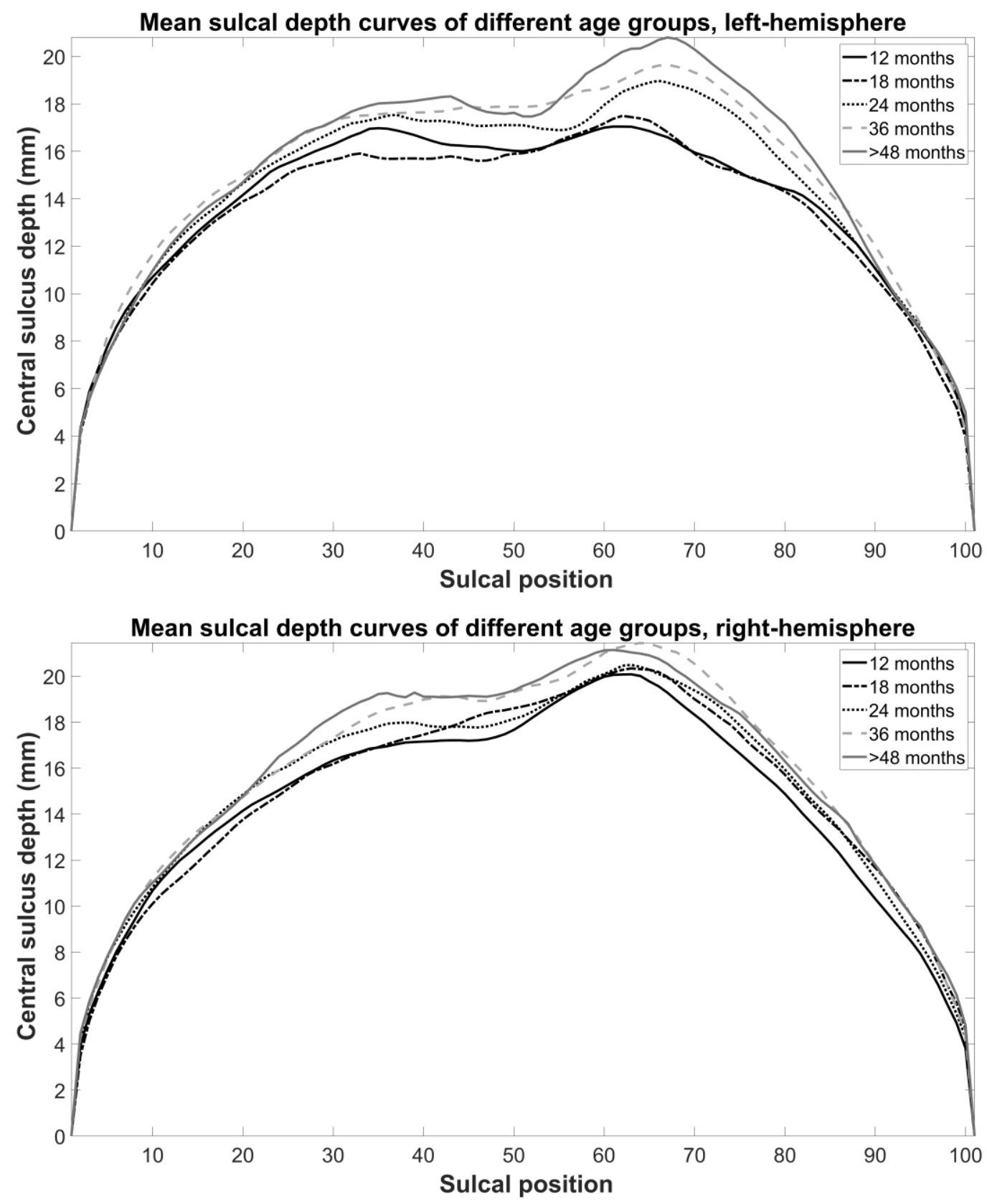

Figure 2. The sulcal depth profiles of the left and right CS for 1,2,3 and 4+ year old groups. Top and Bottom: Left CS and right CS depth curves respectively. 

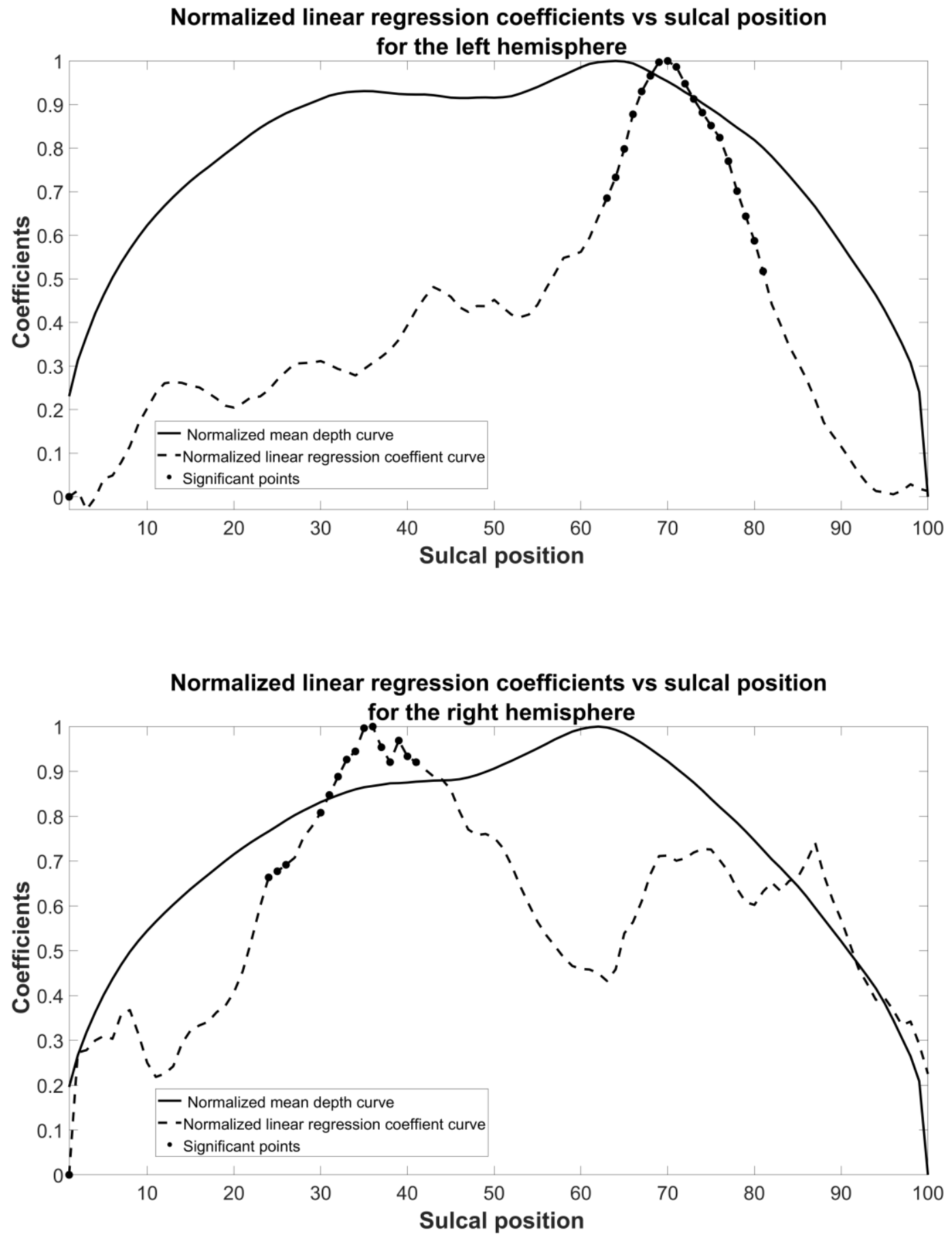

Figure 3. Normalized mean depth curves for all subjects (solid line) and normalized logarithmic regression coefficients for the left CS (top) and right CS (bottom). The higher values indicate greater change. The dots indicate significance after Bonferroni correction. 

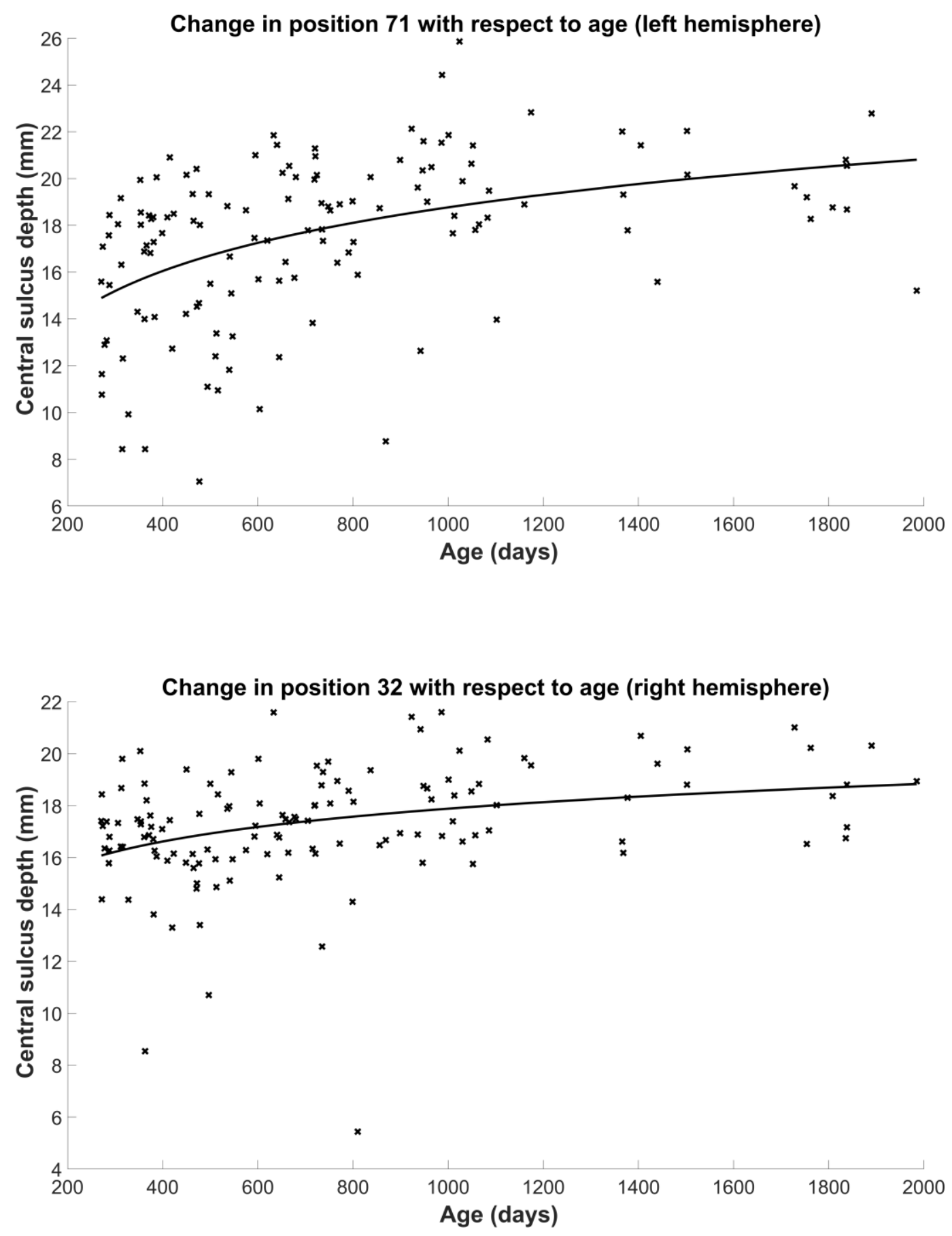

Figure 4. Logarithmic curves fitted to position 71 for the left CS (top) and position 32 for the right CS (bottom). These positions correspond to the areas of greatest change in the regression.

Fig. 5 shows the Mann-Whitney Wilcoxon tests results after correcting for multiple comparisons. This was done to determine the age that drives the changes in the CS. The most significant differences 
seen were between 18 and 24 months in positions 20-40 and 60-80 in the left CS, while in the right hemisphere seen between 12 and 18 months in positions 70-95, between 18 and 24 months in positions 10-30, and positions 38-45 and 60-75 between 24 and 36 months groups. No significant differences were seen after 36 months implying that most changes happen prior to 3 years of age. The results of the significant CS positions in Fig. 5 are summarized in Table 2, along with the corresponding approximate functional areas.

\begin{tabular}{|l|l|l|l|}
\hline & $12 \mathrm{~m} v 18 \mathrm{~m}$ & $18 \mathrm{~m} v 24 \mathrm{~m}$ & $24 \mathrm{~m} v 36 \mathrm{~m}$ \\
\hline Left CS position & & $\begin{array}{l}\text { 24-43: trunk } \\
\text { 65-80: orofacial }\end{array}$ & $70-75:$ orofacial \\
\hline Right CS position & 85-95: orofacial & 10-30: trunk & $\begin{array}{l}38-45: \text { trunk } \\
62-75: \text { orofacial }\end{array}$ \\
\hline
\end{tabular}

Table 2. Table showing positions where Mann-Whitney test results were significant and the corresponding age groups. 

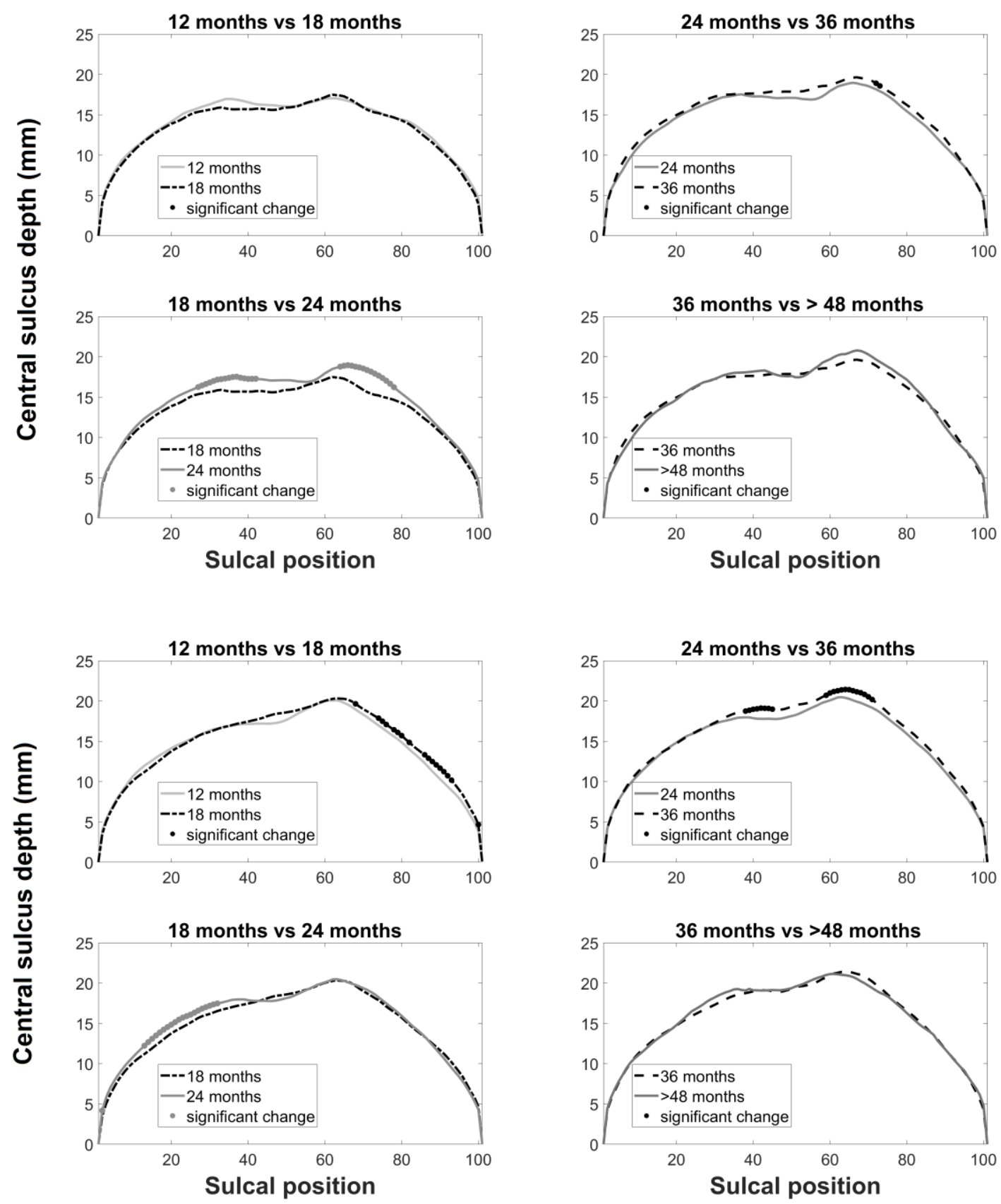

Figure 5. Mann-Whitney test results comparing two groups with 6 months intervals for the left CS (top) and right CS (bottom). The dots indicate regions with significant difference. 


\section{Coefficients of asymmetry for each age group}
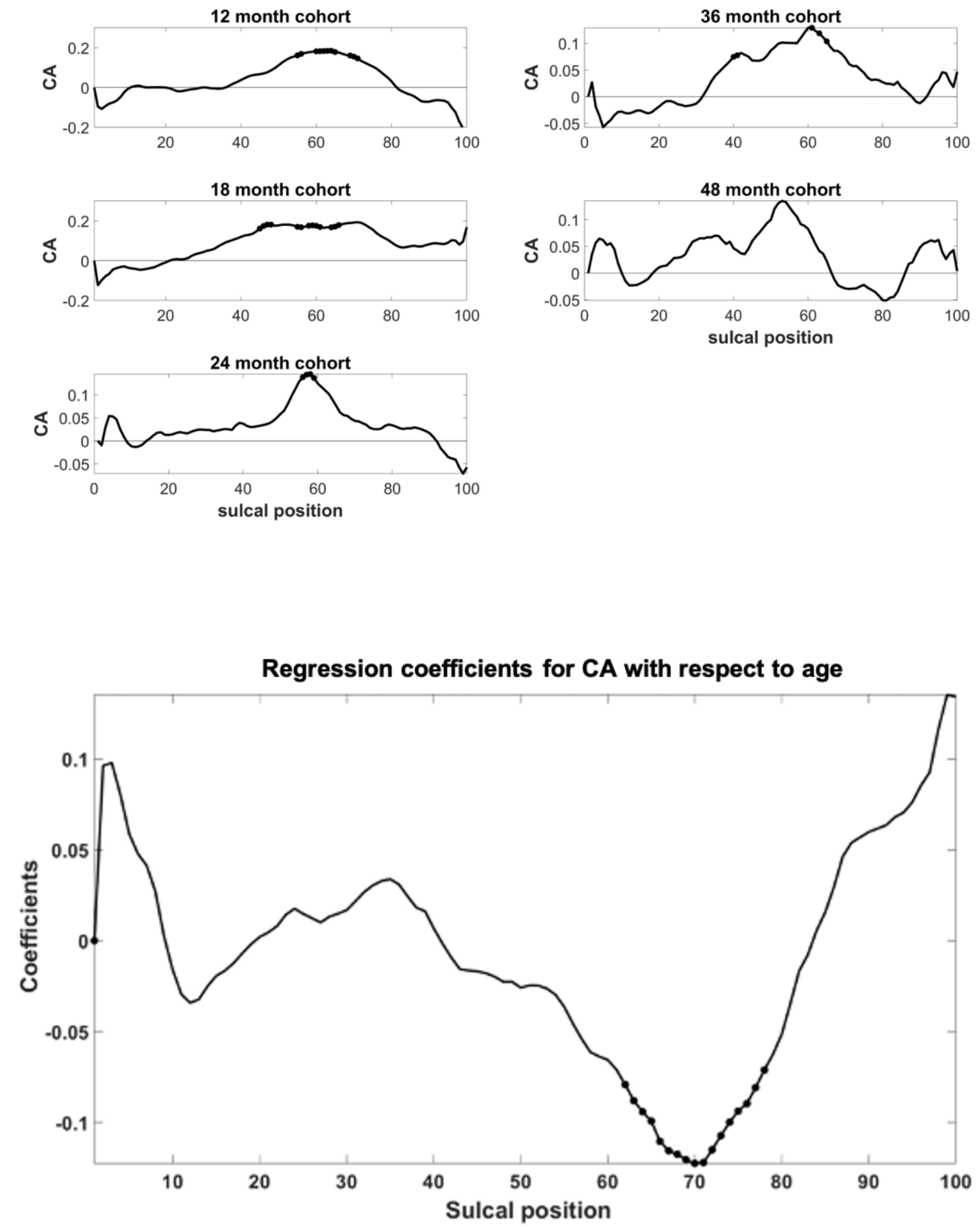

Figure 6. (Top) CA with respect to sulcal position for each age group with regions of significant changes with respect to the next age group in dots. (Bottom) Logarithmic regression coefficient for CA changes over time. The dots indicate region of the CS that showed significant change (uncorrected p-value <0.05) in CA with respect to age. 


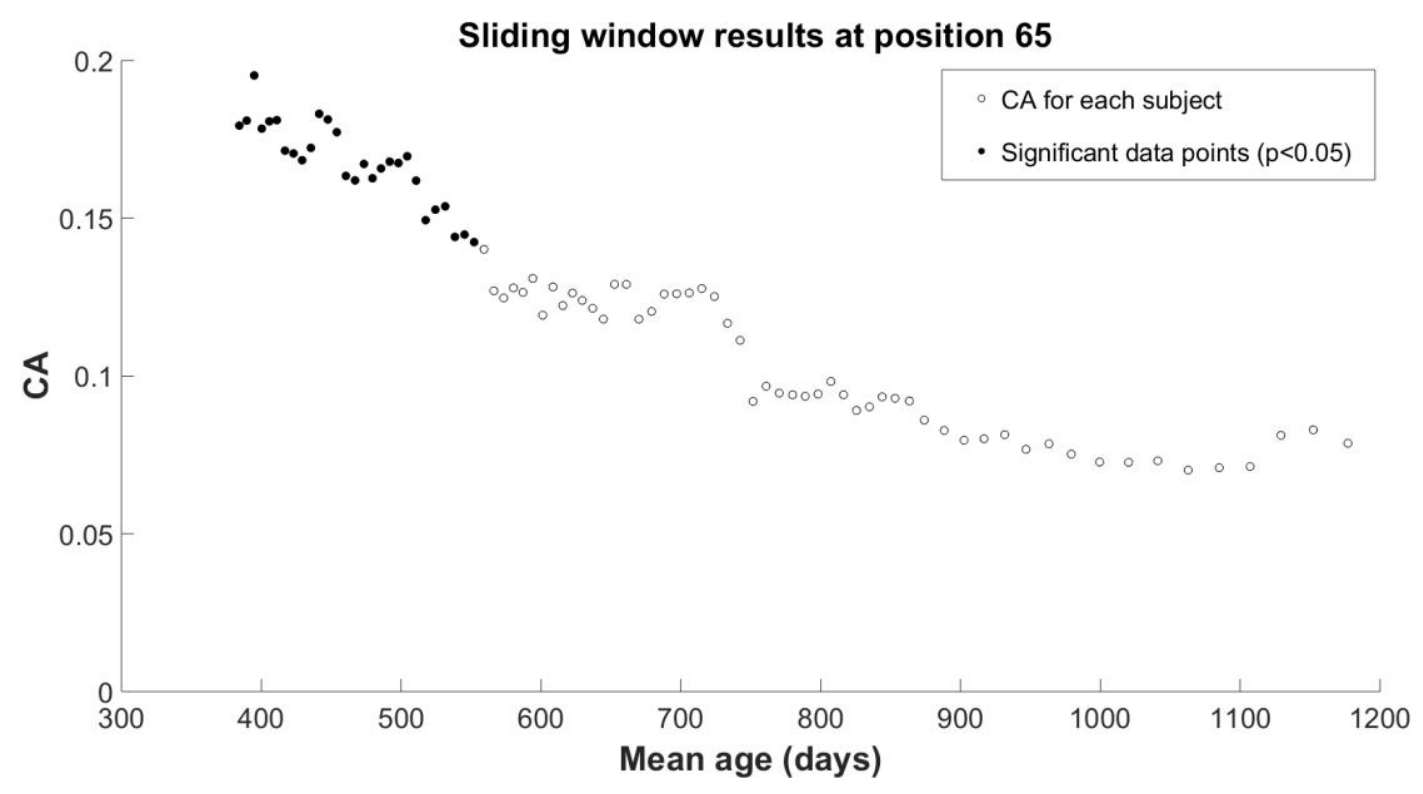

Figure 7. Sliding window analysis result showing asymmetry coefficient with age, at position 65 , and ages of significant changes (black circles). Significant changes occur before day 550, i.e. 18 months of age.

Structural asymmetries are often studied because they can sometimes be associated to the lateralization of brain functions, or to functional specificities. For instance, in the CS, several asymmetries have been associated to manual preference (Coulon et al. 2015; Sun et al., 2012). Here, negative CA values indicate leftward asymmetry. Fig. 6 (top) shows CA in each age group, where the dots indicate positions of significant change with the next age group (Bonferroni corrected $\mathrm{p}$ values at $\mathrm{p}<0.05$ ). It can be observed that there are some significant regions around position 60 before age 5 .

To investigate the relationship between CA and age, we applied logarithmic regression to the CA. The bottom image in Fig. 6 shows the regression coefficients for each position of the CS after applying logarithmic regression with respect to age. Similar to the regression of the left hemisphere sulcal depth, significant CA changes (uncorrected p-value $<0.05$, p-value range: $0-0.0318$ ) were observed in the positions 60-80. The negative coefficient values indicate a decrease of the rightward asymmetry with age. 
Sliding window analysis, which was done to explore how age is involved in the evolution of asymmetry, indicated significance for positions 53-68. Most of the change was focused in the early ages, under 550 days of age, roughly corresponding to 18 months of age. Fig. 7 shows a sample progression of the sliding window for CS position 65. Overall, these results indicate a rightward asymmetry of depth between positions 60 and 80 that decreases with time, disappearing before the age of two years old.

\section{DisCUSSION}

In this paper, we investigated developmental changes of the CS morphology between 12 and 60 months old, in a largely cross-sectional dataset of healthy children. Our analysis shows the CS depth curves at different ages, CS depth asymmetry, comparison between different age groups, and locations of most significant changes. Interpretation of these results may help to understand the link between anatomical development and the emergence of specific functions and pave the way for further research into development of individual sulci.

Studying the depth profile provides insight into the development of the CS and associated functions in early childhood. As age progresses there is a steady increase in CS depth, especially until 36 months (Fig. 2). The CS depth changes are impacted by overall cortical thickness as well as surface area changes, consistent with existing literature indicating increase in brain volume and thickness in the first two years of life (G. Li, Wang, et al. 2014; Knickmeyer et al. 2008). The general shape of the CS depth profile in all age groups is similar to that of adults (Cykowski et al. 2008), thus implying that this shape and a preliminary dip location exist from a very young age. This dip around position 50 in Fig. 2 is known as the 'Pli de Passage Fronto-parietal Moyen' (PPFM), a buried gyrus connecting the frontal and parietal lobes through the CS. The PPFM is an anatomical landmark to hand motor and sensory function (W. W. Boling and Olivier 2004; W. Boling et al. 1999; O. Coulon et al. 2011). Cortical stimulation studies have confirmed the existence of a whole hand motor and sensory functional area at the anatomical location of the PPFM (W. Boling and Olivier 2016) in addition to individual finger and thumb function found more inferiorly along the precentral and postcentral gyri. Furthermore, hand 
motor function was also found in fMRI studies (Yousry et al. 1997). In our dataset, the mean PPFM location for the left hemisphere was at position 50.3 and at 47.1 for the right hemisphere. In order to test if the dip associated to the PPFM emerged after birth, we also calculated the ratio (PPFM/maximum depth of the CS) and its relationship to age. We found no correlation here, which may imply that the PPFM as a morphological feature exists at birth or gets defined earlier than 12 months of age.

Next, we used regression to evaluate which positions of the CS changed most with respect to age. The largest change occurs in between positions 65-80 for the left CS and positions 30-45 for the right CS (Fig. 3). The change in the left hemisphere is likely language related, involving the mouth and tongue region as indicated by the somatosensory homunculus(Catani 2017), while changes in the right hemisphere are indicative of association with the trunk. The differences between the left and right hemispheres may stem from hemispheric asymmetry and variation in GM maturation processes between the hemispheres (Gogtay et al. 2004). In the left hemisphere, the index with the largest change was position 71 of the CS, corresponding to orofacial region. When sulcal depth of this particular position was plotted with respect to age, the curve that emerged mirrored the trend seen in surface area variation of the left precentral gyrus, demonstrated in a previous study investigating cortical growth in children (Remer et al. 2017). On the right hemisphere, the index showing the largest change was position 32, which corresponds to the trunk or hand region. Both left and right CS depth curves in Fig. 4 follow logarithmic changes, as seen in several surface area trajectories of the cortex (Remer et al. 2017).

On the other hand, to quantify when in normal development the CS changes the most, we compared CS depth between consecutive age groups (Fig. 5) and summarized in Table 2. The CS depth changes between age groups vary between the hemispheres with some overlap (i.e. comparing 18 months vs 24 months and 24 months vs 36 months). This difference between the hemispheres may be due to the varying rates of development of the cortical thickness, volume, and surface area (Gogtay et al. 2004). The regions displaying most significance correspond to the upper body areas such as the trunk (positions: 2035 ) and orofacial regions (positions: 65-80). 
According to studies on orofacial control, a sequential development process happens for oral structure control prenatally (Herring 1985), while orofacial control for chewing, sucking and speech production are developed postnatally (Moore and Ruark 1996; Moore, Smith, and Ringel 1988). The jaw movement rather than lip movement is predominant in speech production in the early ages (MacNeilage and Davis 1990). A study investigating speech development in children has shown that jaw control during speech is similar to that of adults by about 15 months of age (Green et al. 2000). The same study, investigating 2 year old speech patterns, hypothesized that independent control of the upper and lower lips are necessary for speech motor development (Green et al. 2000). Children go through multiple stages of vocalization (Ferguson, Menn, and Stoel-Gammon 1992; Oller et al. 1992; Oller 1978; Stark 1980; StoelGammon and Cooper 1984; Vihman and Miller 1988), which include reflexive sounds such as coughing (0-2 months), cooing (1-4 months), expansion, which consists of yelling and screaming and happens along with repetitive limb movements in infants (3-8 months), canonical babbling, which consists of speech patterns with one vowel and consonant and is thought to be associated with jaw oscillatory movement patterns and high involvement of the left hemisphere (J.L. Locke et al. 1995), and meaningful speech (10-18 months)(Eilers et al. 1993; Ejiri 1998; Stark 1980). The 18 months mark, during which meaningful speech production ensues is a key period that repeatedly surfaces in literature. This time frame is also reported to show lexical spurts (John L. Locke 1997), where the number of words acquired per week quadruples. Speech perception studies have shown vocabulary differences to be most prominent between 18 and 24 months (Kuhl et al. 2005). Therefore, there is a strong implication that language acquisition plays a big role in the variations we see.

In the right hemisphere, locations 10-30 associated with the trunk and legs also change significantly (Fig. 5). This may indicate the beginning of lateralization in a sequence of events that may correspond to motor development.

We also investigated how age influences variability in asymmetry by studying the CA and observed rightward asymmetry in most positions of the CS, with significant regions marked in dots (Fig. 6). Additionally, a regression analysis performed on the CA showed largest variation in asymmetry in 
positions 60-80 (marked with dots and corresponding to the language regions in Fig. 3-top). The negative regression coefficients indicate a decrease in rightward asymmetry in this orofacial region. The significance shown with $\mathrm{p}<0.05$ is uncorrected. Although our results were not significant after multiple comparison correction, we believe this trend seen here is relevant to the transforming asymmetry.

There is limited literature investigating cortical measures in children. A previous study (Remer et al. 2017) showed leftward asymmetry of cortical thickness in the precentral gyrus.. The authors also showed a gradual decrease of cortical thickness in the left precentral gyrus and increasing volume in the right pre-central gyrus (wide U-shaped trajectory), which may be imply rightward asymmetry as seen in our analysis. In another study (Gogtay et al. 2004) showed maturation of the cortex by measuring GM loss in subjects of ages 5-20. While GM volume increases at earlier ages and decreases around puberty, the authors found that the left prefrontal cortex showed GM loss in the precentral gyrus at an earlier age, indicating earlier maturation than the right, due to the majority of subjects being right-handed in their study. While handedness information was unavailable for our study, if we assume a larger percentage of children are right-handed, due to prevalence, the rightward asymmetry in Fig. 6 is understandable.

Asymmetry of the CS has been mapped out in adults (Cykowski et al. 2008; Amunts et al. 1996) and leftward asymmetry has been shown in right-handed subjects in the superior extent, close to where we see the largest change in asymmetry in our study. We used a sliding window analysis to tease out the age at which this asymmetry emerges and found a downward trend in the CA with respect to age (example shown in Fig. 7). Similar decreasing trends of the rightward asymmetry were also seen in positions 55-66, with significance generally seen at ages under 24 months. The negative trend may indicate the emergence of handedness. We also tested brain hemispheric volume instead of age as the dependent variable in our regression model. However, this did not show as strong a relationship as with age. While age and brain volume are correlated, (correlation coefficient=0.6), that the PPFM location did not correlate with volume perhaps indicates that the CS development reflects more the functional skills rather than simply brain volume (i.e. a younger child with larger brain volume would not achieve the 
same developmental milestones as an older child with a smaller brain volume). Future studies involving correlation of motor and language scores with the PPFM will facilitate a deeper understanding here.

Finally, it has been shown that in the adult CS, there are three types of distributions of the CS; unimodal, bimodal and trimodal (Cykowski et al. 2008). Bimodal distributions, which have clear inferior and superior peaks with a dip corresponding to the PPFM, were the most common with $94.6 \%$ of the left CS and $80 \%$ of the right CS. However, $3.6 \%$ of the left CS and $16.4 \%$ of the right CS were shown to be trimodal, while $1.8 \%$ of the left CS and $3.6 \%$ of the right CS were shown to be unimodal (Cykowski et al. 2008). In our dataset, while bimodal was the most common distribution at $77 \%$ for the left CS and $62 \%$ for the right CS, $13.7 \%$ of the left CS and $29 \%$ of the right CS was unimodal, and $10 \%$ of the left CS and 9\% of the right CS was trimodal. There is a clear similarity with the adult CS in that the bimodal distribution is most common. However, the percentages of the unimodal and trimodal distributions were much higher in our data, perhaps indicating that these distributions change post 60 months as the child grows into adulthood.

A limitation in our study is the uneven number of subjects in individual age groups. Our dataset includes more subjects in the younger ages and therefore our sample sizes are not evenly distributed. This makes it difficult to account for individual variability in the data. Although our data included both genders, investigating gender-based differences was not possible due to too small a sample size for each gender within each age group. Additionally, due to most of our data being cross-sectional, it was not possible to declare if and when the CS distributions change, for example if a child who had a unimodal distribution at a younger age changed to a bimodal distribution as they became older. However, considering the small percentage of adults who have unimodal distributions, the CS shape may well change over time to become more bimodal. A follow-up with longitudinal data will enable us to understand the change in the CS distribution more accurately. 


\section{CONCLUSION}

In this study, we investigated CS changes with respect to age in early childhood. We characterized the CS morphology and inter-hemispheric asymmetries at various ages between 12 and 60 months old children, and found a potential relationship between anatomical changes and language development, especially at around 18 months of age, which is a critical period for language. Due to the variations in individual children in development, more subjects need to be assessed to improve the accuracy of our findings in future studies. Additionally, longitudinal MRI data, genetic information and handedness will also provide more insight into characterization of the CS.

\section{REFERENCES}

Amunts, Katrin, Lutz Jäncke, Hartmut Mohlberg, Helmuth Steinmetz, and Karl Zilles. 2000. "Interhemispheric Asymmetry of the Human Motor Cortex Related to Handedness and Gender." Neuropsychologia 38 (3): $304-12$.

Amunts, Katrin, Gottfried Schlaug, Axel Schleicher, Helmuth Steinmetz, Andreas Dabringhaus, Per E. Roland, and Karl Zilles. 1996. "Asymmetry in the Human Motor Cortex and Handedness." NeuroImage 4 (3): $216-22$.

Andersen, Simon Kragh, Christian Elmholt Jakobsen, Claus Hougaard Pedersen, Anders Munk Rasmussen, Maciej Plocharski, and Lasse Riis Østergaard. n.d. “Classification of Alzheimer’s Disease from MRI Using Sulcal Morphology.”

Boling, Warren, and André Olivier. 2016. “Anatomy of Important Functioning Cortex.” In Functional Mapping of the Cerebral Cortex, 23-40. Cham: Springer International Publishing.

Boling, Warren, André Olivier, Richard G. Bittar, and David Reutens. 1999. “Localization of Hand Motor Activation in Broca's Pli de Passage Moyen.” Journal of Neurosurgery 91 (6): 903-10.

Boling, Warren W, and André Olivier. 2004. "Localization of Hand Sensory Function to the Pli de Passage Moyen of Broca."

Brain Development Cooperative Group, Brain Development Cooperative. 2012. "Total and Regional Brain Volumes in a Population-Based Normative Sample from 4 to 18 Years: The NIH MRI Study of Normal 
Brain Development." Cerebral Cortex (New York, N.Y. : 1991) 22 (1): 1-12.

Catani, Marco. 2017. “A Little Man of Some Importance.” Brain 140 (11): 3055-61.

Coulon, O., F. Pizzagalli, G. Operto, G. Auzias, C. Delon-Martin, and M. Dojat. 2011. "Two New Stable Anatomical Landmarks on the Central Sulcus: Definition, Automatic Detection, and Their Relationship with Primary Motor Functions of the Hand.” In 2011 Annual International Conference of the IEEE Engineering in Medicine and Biology Society, 2011:7795-98. IEEE.

Coulon, Olivier, Julien Lefevre, Stefan Kloppel, Hartwig Siebner, and Jean-Francois Mangin. 2015. “QuasiIsometric Length Parameterization of Cortical Sulci: Application to Handedness and the Central Sulcus Morphology.” In 2015 IEEE 12th International Symposium on Biomedical Imaging (ISBI), 1268-71. IEEE.

Courchesne, Eric, Heather J. Chisum, Jeanne Townsend, Angilene Cowles, James Covington, Brian Egaas, Mark Harwood, Stuart Hinds, and Gary A. Press. 2000. "Normal Brain Development and Aging: Quantitative Analysis at in Vivo MR Imaging in Healthy Volunteers.” Radiology 216 (3): 672-82.

Cykowski, Matthew D., Olivier Coulon, Peter V. Kochunov, Katrin Amunts, Jack L. Lancaster, Angela R. Laird, David C. Glahn, and Peter T. Fox. 2008. "The Central Sulcus: An Observer-Independent Characterization of Sulcal Landmarks and Depth Asymmetry." Cerebral Cortex 18 (9): 1999-2009.

Deoni, Sean C.L., Brian K. Rutt, and Terry M. Peters. 2006. "Synthetic T1-Weighted Brain Image Generation with Incorporated Coil Intensity Correction Using DESPOT1.” Magnetic Resonance Imaging 24 (9): $1241-48$.

Deoni, Sean C L, Brian K Rutt, and Terry M Peters. 2003. "Rapid Combined T1 and T2 Mapping Using Gradient Recalled Acquisition in the Steady State." Magnetic Resonance in Medicine 49 (3): 515-26.

Eilers, Rebecca E., D. Kimbrough Oller, Sharyse Levine, Devorah Basinger, Michael P. Lynch, and Richard Urbano. 1993. "The Role of Prematurity and Socioeconomic Status in the Onset of Canonical Babbling in Infants." Infant Behavior and Development 16 (3): 297-315.

Ejiri, K. 1998. "Relationship between Rhythmic Behavior and Canonical Babbling in Infant Vocal Development." Phonetica 55 (4): 226-37.

Evans, Alan C. 2006. “The NIH MRI Study of Normal Brain Development.” NeuroImage 30 (1): 184-202. 
Ferguson, Charles A. (Charles Albert), Lise. Menn, and Carol. Stoel-Gammon. 1992. Phonological Development: Models, Research, Implications. Timonium Md.: York Press.

Fischer, C, G Operto, S Laguitton, M Perrot, I Denghien, D Riviere, and J.-F. Mangin. 2012. “Morphologist 2012: The New Morphological Pipeline of BrainVISA.” In Proc. HBM.

Galaburda, Albert M., J. Eric Schmitt, Scott W. Atlas, Stephan Eliez, Ursula Bellugi, and Allan L. Reiss. 2001. “Dorsal Forebrain Anomaly in Williams Syndrome.” Archives of Neurology 58 (11): 1865.

Gaonkar, Bilwaj, Luke Macyszyn, Michel Bilello, Mohammed Salehi Sadaghiani, Hamed Akbari, Mark A Attiah, Zarina S Ali, et al. 2015. "Automated Tumor Volumetry Using Computer-Aided Image Segmentation." Academic Radiology 22 (5): 653-61.

Giedd, Jay N., and Judith L. Rapoport. 2010. "Structural MRI of Pediatric Brain Development: What Have We Learned and Where Are We Going?" Neuron 67 (5): 728-34.

Gilles, F. H., and Marvin D. Nelson. 2012. The Developing Human Brain : Growth and Adversities. Mac Keith Press.

Gogtay, Nitin, Jay N Giedd, Leslie Lusk, Kiralee M Hayashi, Deanna Greenstein, A Catherine Vaituzis, Tom F Nugent, et al. 2004. "Dynamic Mapping of Human Cortical Development during Childhood through Early Adulthood." Proceedings of the National Academy of Sciences of the United States of America 101 (21): 8174-79.

Green, J R, C A Moore, M Higashikawa, and R W Steeve. 2000. “The Physiologic Development of Speech Motor Control: Lip and Jaw Coordination." Journal of Speech, Language, and Hearing Research : JSLHR 43 (1): 239-55.

Herring, Susan W. 1985. “The Ontogeny of Mammalian Mastication.” American Zoologist 25 (2): 339-50. Hill, Jason, Donna Dierker, Jeffrey Neil, Terrie Inder, Andrew Knutsen, John Harwell, Timothy Coalson, and David Van Essen. 2010. “A Surface-Based Analysis of Hemispheric Asymmetries and Folding of Cerebral Cortex in Term-Born Human Infants.” Journal of Neuroscience 30 (6).

Hopkins, W.D., O. Coulon, and J.-F. Mangin. 2010. “Observer-Independent Characterization of Sulcal Landmarks and Depth Asymmetry in the Central Sulcus of the Chimpanzee Brain.” Neuroscience 171 (2): 544-51. 
Jackowski, Andrea P, and Robert T. Schultz. 2005. "Foreshortened Dorsal Extension of the Central Sulcus in Williams Syndrome.” Cortex 41 (3): 282-90.

Jenkinson, M, and S Smith. 2001. “A Global Optimisation Method for Robust Affine Registration of Brain Images." Medical Image Analysis 5 (2): 143-56.

Jenkinson, Mark, Peter Bannister, Michael Brady, and Stephen Smith. 2002. "Improved Optimization for the Robust and Accurate Linear Registration and Motion Correction of Brain Images." NeuroImage 17 (2): $825-41$.

Keshavan, Matcheri S, Vaibhav A Diwadkar, Michael DeBellis, Elizabeth Dick, Rupali Kotwal, David R Rosenberg, John A Sweeney, Nancy Minshew, and Jay W Pettegrew. 2002. "Development of the Corpus Callosum in Childhood, Adolescence and Early Adulthood.” Life Sciences 70 (16): 1909-22.

Knickmeyer, Rebecca C, Sylvain Gouttard, Chaeryon Kang, Dianne Evans, Kathy Wilber, J Keith Smith, Robert M Hamer, Weili Lin, Guido Gerig, and John H Gilmore. 2008. “A Structural MRI Study of Human Brain Development from Birth to 2 Years.” The Journal of Neuroscience : The Official Journal of the Society for Neuroscience 28 (47): 12176-82.

Kuhl, Patricia K, Barbara T Conboy, Denise Padden, Tobey Nelson, and Jessica Pruitt. 2005. "Early Speech Perception and Later Language Development: Implications for the 'Critical Period."' Language Learning and Development 1 (3-4): 237-64.

Lenroot, Rhoshel K., and Jay N. Giedd. 2006. "Brain Development in Children and Adolescents: Insights from Anatomical Magnetic Resonance Imaging.” Neuroscience \& Biobehavioral Reviews 30 (6): 718-29.

Li, Gang, Jingxin Nie, Li Wang, Feng Shi, John H. Gilmore, Weili Lin, and Dinggang Shen. 2014a. "Measuring the Dynamic Longitudinal Cortex Development in Infants by Reconstruction of Temporally Consistent Cortical Surfaces.” NeuroImage 90: 266-79.

Li, Gang, Li Wang, Feng Shi, Amanda E Lyall, Weili Lin, John H Gilmore, and Dinggang Shen. 2014b. "Mapping Longitudinal Development of Local Cortical Gyrification in Infants from Birth to 2 Years of Age.” The Journal of Neuroscience : The Official Journal of the Society for Neuroscience 34 (12): 422838. 
Li, Shuyu, Ying Han, Deyi Wang, Hong Yang, Yubo Fan, Yating Lv, Hehan Tang, Qiyong Gong, Yufeng Zang, and Yong He. 2010. "Mapping Surface Variability of the Central Sulcus in Musicians.” Cerebral Cortex 20 (1): 25-33.

Li, Shuyu, Shaoyi Wang, Xinwei Li, Qiongling Li, and Xiaobo Li. 2015. “Abnormal Surface Morphology of the Central Sulcus in Children with Attention-Deficit/Hyperactivity Disorder." Frontiers in Neuroanatomy 9 (August): 114.

Li, Shuyu, Mingrui Xia, Fang Pu, Deyu Li, Yubo Fan, Haijun Niu, Baoqing Pei, and Yong He. 2011. “AgeRelated Changes in the Surface Morphology of the Central Sulcus."

Locke, J.L., K.E. Bekken, L. Mcminnlarson, and D. Wein. 1995. "Emergent Control of Manual and VocalMotor Activity in Relation to the Development of Speech.” Brain and Language 51 (3): 498-508.

Locke, John L. 1997. “A Theory of Neurolinguistic Development.” Brain and Language 58 (2): 265-326. Lyall, Amanda E., Feng Shi, Xiujuan Geng, Sandra Woolson, Gang Li, Li Wang, Robert M. Hamer, Dinggang Shen, and John H. Gilmore. 2015. "Dynamic Development of Regional Cortical Thickness and Surface Area in Early Childhood." Cerebral Cortex 25 (8): 2204-12.

MacNeilage, Peter F., and Barbara L. Davis. 1990. “Acquisition of Speech Production: The Achievement of Segmental Independence.” In Speech Production and Speech Modelling, 55-68. Dordrecht: Springer Netherlands.

McKay, D Reese, Peter Kochunov, Matthew D Cykowski, Jack W Kent, Angela R Laird, Jack L Lancaster, John Blangero, David C Glahn, and Peter T Fox. 2013. "Sulcal Depth-Position Profile Is a Genetically Mediated Neuroscientific Trait: Description and Characterization in the Central Sulcus.” The Journal of Neuroscience: The Official Journal of the Society for Neuroscience 33 (39): 15618-25.

Moore, Christopher A., and Jacki L. Ruark. 1996. "Does Speech Emerge From Earlier Appearing Oral Motor Behaviors?” Journal of Speech Language and Hearing Research 39 (5): 1034.

Moore, Christopher A., Anne Smith, and Robert L. Ringel. 1988. "Task-Specific Organization of Activity in Human Jaw Muscles.” Journal of Speech Language and Hearing Research 31 (4): 670.

Nie, Jingxin, Gang Li, and Dinggang Shen. 2013. "Development of Cortical Anatomical Properties from Early Childhood to Early Adulthood.” NeuroImage 76 (August): 216-24. 
Oller, D Kimbrough. 1978. "Infant Vocalization and the Development of Speech.” Allied Health and Behavioral Sciences 1 (4): 523-49.

Oller, D Kimbrough, Michael P Lynch, C A Ferguson, L Menn, and C Stoel-Gammon. 1992. “Infant Vocalizations and Innovations in Infraphonology: Toward a Broader Theory of Development and Disorders." Phonological Development: Models, Research, Implications, 509-36.

Penfield, Wilder, and Theodore Rasmussen. 1950. The Cerebral Cortex of Man; a Clinical Study of Localization of Function. The Cerebral Cortex of Man; a Clinical Study of Localization of Function. Oxford, England: Macmillan.

Perrot, Matthieu, Denis Rivière, and Jean-François Mangin. 2011. "Cortical Sulci Recognition and Spatial Normalization." Medical Image Analysis 15 (4): 529-50.

Raznahan, Armin, Phillip Shaw, Francois Lalonde, Mike Stockman, Gregory L Wallace, Dede Greenstein, Liv Clasen, Nitin Gogtay, and Jay N Giedd. 2011. “How Does Your Cortex Grow?” The Journal of Neuroscience: The Official Journal of the Society for Neuroscience 31 (19): 7174-77.

Remer, Justin, Elise Croteau-Chonka, Douglas C. Dean, Sara D'Arpino, Holly Dirks, Dannielle Whiley, and Sean C.L. Deoni. 2017. "Quantifying Cortical Development in Typically Developing Toddlers and Young Children, 1-6 Years of Age.” NeuroImage 153 (June): 246-61.

Roux, Franck-Emmanuel, Imène Djidjeli, and Jean-Baptiste Durand. 2018. "Functional Architecture of the Somatosensory Homunculus Detected by Electrostimulation.” The Journal of Physiology 596 (5): 94156.

Shattuck, David W, and Richard M Leahy. 2002. "BrainSuite: An Automated Cortical Surface Identification Tool." Medical Image Analysis 6 (2): 129-42.

Sowell, Elizabeth R., Bradley S. Peterson, Paul M. Thompson, Suzanne E. Welcome, Amy L. Henkenius, and Arthur W. Toga. 2003. "Mapping Cortical Change across the Human Life Span.” Nature Neuroscience 6 (3): 309-15.

Sowell, Elizabeth R, Paul M Thompson, Christiana M Leonard, Suzanne E Welcome, Eric Kan, and Arthur W Toga. n.d. "Longitudinal Mapping of Cortical Thickness and Brain Growth in Normal Children." Sowell, Elizabeth R, Doris A Trauner, Anthony Gamst, and Terry L Jernigan. 2007. "Development of Cortical 
and Subcortical Brain Structures in Childhood and Adolescence: A Structural MRI Study."

Developmental Medicine \& Child Neurology 44 (1): 4-16.

Stark, Rachel E. 1980. “STAGES OF SPEECH DEVELOPMENT IN THE FIRST YEAR OF LIFE.” Child Phonology, January, 73-92.

Stoel-Gammon, C, and J A Cooper. 1984. "Patterns of Early Lexical and Phonological Development." Journal of Child Language 11 (2): 247-71.

Sun, Z. Y., Klöppel, S., Rivière, D., Perrot, M., Frackowiak, R. S. J., Siebner, H., \& Mangin, J.-F. (2012). The effect of handedness on the shape of the central sulcus. NeuroImage, 60(1), 332-339. https://doi.org/10.1016/j.neuroimage.2011.12.050

Toga, Arthur W, Paul M Thompson, and Elizabeth R Sowell. 2011. "Mapping Brain Maturation.” Trends in Neurosciences 29 (3): 148-59.

Vihman, Marilyn May, and Ruth Miller. 1988. "Words and Babble at the Threshold of Language: Acquisition."

Yousry, T., U D Schmid, H Alkadhi, D Schmidt, A Peraud, A Buettner, and P Winkler. 1997. "Localization of the Motor Hand Area to a Knob on the Precentral Gyrus. A New Landmark.” Brain 120 (1): 141-57. 